\title{
Neue Protokollempfehlungen zu Mess-Sequenzen der MRT der Wirbelsäule
}

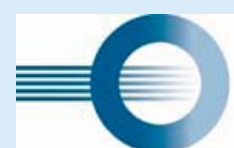

AG Bildgebende Verfahren des Bewegungsapparates

in der Deutschen Röntgengesellschaft

Die AG Bildgebende Verfahren des Bewegungsapparates der Deutschen Röntgengesellschaft (AG BVB) erarbeitet zu allen wesentlichen Untersuchungen der muskuloskelettalen Radiologie Protokollempfehlungen, die ein qualitativ hochwertiges Untersuchungsergebnis und einen aussagekräftigen Befund gewährleisten und dabei die Erfordernisse der Routine in der Klinik und in der niedergelassenen Radiologie berücksichtigen. Frau Prof. Dr. Andrea Baur-Melnyk vom Klinikum Großhadern, München, und im Vorstand der AG BVB zuständig für strukturierte Befundung erläutert im Interview die aktuellen Protokollempfehlungen zu Mess-Sequenzen der MRT der Wirbelsäule.

Frau Professor Baur-Melnyk, Rückenschmerzen sind heute eine echte Volkskrankheit. Welche Rolle spielt hier die bildgebende Diagnostik?

Fangen wir mit ein paar Zahlen an: 85 Prozent aller Menschen in Deutschland leiden einmal im Leben an Rückenschmerz. 15 bis 22 Prozent leiden zudem an chronischem beziehungsweise chronisch-rezidivierendem Rückenschmerz. Für 31 Prozent aller Krankheitstage ist Rückenschmerz die Ursache sowie für 50 Prozent aller gestellten Rentenanträge. Die Gesamtkosten aufgrund von Rückenschmerz belaufen sich auf etwa 8 Milliarden Euro pro Jahr in Deutschland. Das Thema ist sozioökonomisch also extrem wichtig. Bei der Diagnostik von Rückenschmerz sollte die Bildgebung nicht automatisch erfolgen. So ist bei akutem beziehungsweise unspezifischem Rückenschmerz zunächst keine Bildgebung durchzuführen, weil nach aktuellen Studien dadurch hohe Kosten und Folgekosten entstehen, häufiger Chronifizierungen auftreten und auch unnötige Eingriffe durchgeführt werden. Bei akutem Rückenschmerz kommt die Bildgebung nur bei den sogenannten „Red Flags“ zum Einsatz. Red Flags sind Begleitsymptome und Vorerkrankungen, die als Warnsignal für eine spezifische
Ursache mit dringendem Handlungsbedarf stehen. Beim Rückenschmerz heißt das, wenn man Hinweise hat auf eine Fraktur, einen Tumor, eine Infektion oder radikuläre Symptome. Des Weiteren sollte eine Bildgebung bei subakuten oder chronischen Rückenschmerzen erfolgen, wenn nach 6 Wochen leitliniengerechter Therapie kein Erfolg erzielt wurde. Grundlage hierfür ist die Leitlinie „Nationale VersorgungsLeitlinie Kreuzschmerz“ von AWMF, BÄK und KBV.

\section{In welchen Fällen kommt die MRT bei der Diagnostik zum Einsatz?}

Bei radikulären Symptomen, bei Infektionen und bei Tumoren ist eine MRT sinnvoll und hier kommt sie auch in der Regel zum Einsatz. Die häufigste radikuläre Symptomatik ist die Bandscheiben-Herniation. Seltenere Ursachen, die die MRT auch aufklären kann, sind synoviale Zysten, aber auch Tumoren oder Metastasen, die sich in der Wirbelsäule manifestiert haben. Eine nicht radikuläre Symptomatik braucht in der Regel keine akute Bildgebung. Nur wenn die schon oben beschriebenen Red Flags vorliegen oder wenn eine leitliniengerechte Therapie zu keiner Besserung geführt hat, kommt auch hier eine MRT zum Einsatz.

Weshalb hat die AG BVB nun Protokollempfehlungen zu Mess-Sequenzen der MRT für die Wirbelsäule veröffentlicht?

Sehr oft ist es schwierig, die genaue Ursache für den Rückenschmerz herauszufinden. Muskuläre Dysbalancen und Fehlhaltungen führen häufig zu Beschwerden; diese können wir im MRT aber nicht sehen. Zudem haben Patienten häufig mehrere Erkrankungen. Oftmals liegen gleichzeitig eine Bandscheiben-Herniation und eine Beckengelenksarthrose vor. Was dann genau den Schmerz verursacht, kann man nur vermuten. Mit den Protokollempfehlungen möchten wir eine Hilfestellung geben, welche Bildgebung für welche Indikation die beste ist und zum möglichst besten Ergebnis führt.

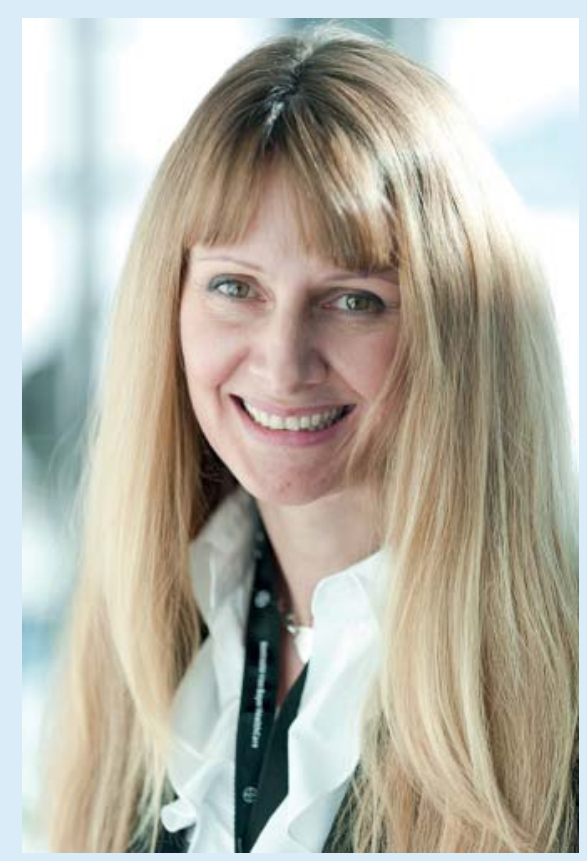

Prof. Dr. Andrea Maur-Melnyk

Welche Indikationen sind in den Protokollempfehlungen abgedeckt?

Wir haben die Empfehlungen in 2 Hauptprotokolle gegliedert: Im Sequenzprotokoll „Wirbelsäule nativ“ geht es um degenerative Wirbelsäulenveränderungen, um Frakturen und somit auch um das große Feld der Bandscheibenproblematik. Im Sequenzprotokoll „Wirbelsäule KM“ geht es um die Sequenzen, in denen ein Kontrastmittel verabreicht wird. Also bei Tumoren und Metastasen sowie bei entzündlichen Veränderungen.

\section{Wie finden die Empfehlungen nun Ein- gang in die klinische Routine?}

Ob diese Empfehlungen in der Praxis auch Anwendung finden, ist natürlich die große Frage. Für unser Haus in Erlangen kann ich sagen, dass diese beiden Protokolle überall abgelegt sind und auch immer so gefahren werden. Unsere Erfahrungen damit sind sehr positiv und wir können es allen Kolleginnen und Kollegen nur ans Herz liegen. 